\title{
Der Tod von Patienten hinterlässt Spuren
}

\begin{abstract}
Stirbt ein Patient, trauern nicht nur die Angehörigen. Sein Tod hinterlässt auch Spuren bei dem behandelnden Arzt. Diese können dessen eigenes Leben, das seiner Familie und das Wohlergehen anderer Patienten beeinflussen.
\end{abstract}

n den Jahren 2010 und 2011 befragte das Autorenteam insgesamt 20 Onkologen mit unterschiedlicher Berufserfahrung aus drei klinischen Zentren in Ontario.

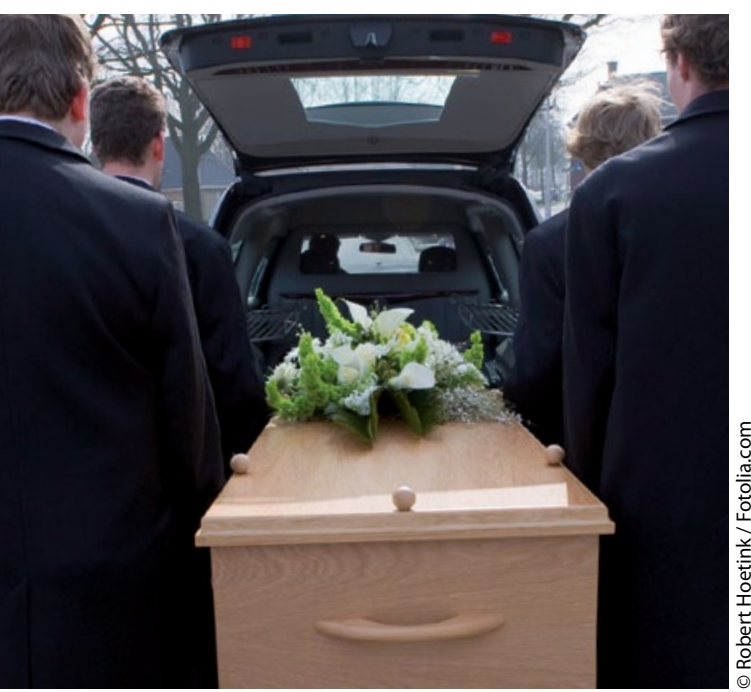

Trauerarbeit trifft nicht nur die Angehörigen - sondern auch den behandelnden Arzt.
$70 \%$ der Befragten gaben Trauer an, teilweise bereits vor dem Ableben des Patienten. Jeder Dritte hatte geweint und mit Schlafproblemen zu kämpfen. Die Ärzte empfanden Verantwortung für das Leben des Patienten und fühlten sich nach dessen Tod machtlos. Es kamen Selbstzweifel sowie Gedanken zu Schuld und Versagen auf.

Die meisten (85\%) bemühten sich, diese Empfindungen von ihrem allgemeinen Leben abzugrenzen. Doch nicht einmal jeder Zweite der befragten Onkologen fühlte sich ausreichend imstande, die Balance zwischen der Nähe zu einem sterbenden Patienten und der nötigen Distanz zu finden.

Manche Mediziner versuchten, sich vor emotionaler Überlastung zu schützen, indem sie sich von einem Patienten und dessen Familie zurückzogen, sobald der Tod näher rückte. Dann wurde der Sterbende beispielsweise immer seltener am Krankenbett besucht.

Die ständige Konfrontation mit dem Tod hatte für fast die Hälfte der Onko- logen allerdings auch positive Aspekte: Sie berichteten beispielsweise darüber, durch diese Erfahrungen eine bessere eigene Lebensperspektive gefunden zu haben.

Fazit: Der Anteil von Medizinern, die durch den Tod ihres Patienten leiden, ist auffallend hoch. In einem Kommentar betonen Michelle Shayne und Timothy E. Quill von der University of Rochester, dass anhaltende Trauer zu einem Burnout beitragen kann. Ärzte müssten Bewältigungsstrategien wie Ausgleich in der Familie, Kontakt mit Freunden oder Interessen außerhalb des Medizinbetriebs entwickeln. Genauso wichtig sei es, die eigene Trauer zu erkennen und mit ihr umzugehen. Mit Blick auf Burn-out-Raten von $56 \%$ bei den Onkologen und immerhin bereits 30\% beim Facharztnachwuchs hat die Universität von Rochester eine Fachgruppe für onkologisch tätiges Personal gegründet. Die Gruppe trifft sich regelmäßig - nicht nur, um sich auszutauschen, sondern auch, um zu meditieren und um kürzlich verstorbene Patienten zu trauern.

Dr. Christine Starostzik

Granek L et al. Nature and impact of grief over patient loss on oncologists' personal and professional lives. Arch Intern Med 2012; 172: 964-66

\section{Nach Verlegen des Estrichs in die Hautklinik}

Der Zementbrand ist in Deutschland offenbar der häufigste Grund für ein schweres irritatives Kontaktekzem. Betroffen sind vor allem Heimwerker.

E ine Autorengruppe um Dr. Heiko Poppe von der Universität Würzburg hat Ursachen und Verlauf von akuten Kontaktekzemen bei 54 Patienten ausgewertet, die in der dortigen dermatologischen Klinik behandelt worden waren.

Am häufigsten war nasser Zement Ursache der Beschwerden (elf Patienten). Es folgten topische Arzneien (zehn Patienten), Desinfektionsmittel (acht Patienten), biologische Flüssigkeiten, z. B. Harn (sieben Patienten) sowie Lösungsmittel (sechs Patienten).

Nasser Zement war das einzige Agens, das Grad-IV-Verbrennungen herbeiführte; diese wurden bei allen elf Zement- brandpatienten, jedoch bei keiner anderen Patientengruppe beobachtet.

Alkalische Mittel, so Poppe und Mitarbeiter, schädigen die Haut oft viel tiefer und schwerer als Säure. Zudem verursachen sie nicht so schnell Schmerzen. Bei den Patienten mit Zementbrand begannen die Symptome mit Hautrötungen und einem brennenden Gefühl meist vier bis acht Stunden nach Exposition. Einen Dermatologen suchten die Heimwerker allerdings erst nach ein bis drei Tagen auf.

Für die erste Hilfe empfehlen die Autoren eine gründliche Reinigung von jeglichen Zementresten mit Wasser, gegebe- nenfalls mit 1\%iger Essigsäure. Bei fortgeschrittenem Zementbrand geben Poppe und Mitarbeiter einer antiseptischen, kühlenden topischen Behandlung den Vorzug und raten gleichzeitig von topischen Antibiotika wegen der möglichen Resistenzentwicklung ab.

Fazit: Die Gefahr durch nassen Zement, so die Studienautoren, wird gerade von Heimwerkern häufig unterschätzt. Im Gegensatz zu professionellen Handwerkern, die im Umgang mit dem Material geschult sind und Schutzkleidung tragen, wissen Heimwerker meist nicht, wie gefährlich Zement sein kann. Thomas Müller

Poppe $\mathrm{H}$ et al. Do-it-yourself cement work: the main cause of severe irritant contact dermatitis requiring hospitalization. Contact Dermatitis 2012 Aug 20; Epub ahead of print 